\title{
Arquitectura Web para análisis de sentimientos en Facebook con enfoque semántico
}

\author{
Carlos Acevedo Miranda ${ }^{1}$, Ricardo Clorio Rodriguez ${ }^{1}$, Roberto Zagal Flores ${ }^{1}$, \\ and Consuelo V. García Mendoza ${ }^{1}$ \\ ${ }^{1}$ Escuela Superior de Cómputo, Instituto Politécnico Nacional, \\ Ciudad de México, México \\ carls.9204@gmail.com, ricardo-clorio@hotmail.com, zagalmmx@gmail.com, \\ varinia400@hotmail.com \\ http://www.escom.ipn.mx
}

\begin{abstract}
Resumen Actualmente el potencial del análisis automático de sentimientos en redes sociales es relevante en el desarrollo de aplicaciones para múltiples propósitos. En este sentido, las publicaciones en Facebook escritas en español pueden contener información semántica relevante que permitirían clasificarlas en categorías de emociones, la complejidad del idioma hace que el análisis de esta información sea difícil y dependa de identificar palabras que tengan significado emocional. En este sentido, proponemos un enfoque que integra una aplicación web, una jerarquía semántica basada en WordNet-Affect Hierachy en español y un clasificador Naive Bayes que identifica las publicaciones en las emociones alegría, tristeza y enojo.. Los resultados experimentales muestran una precisión del $63 \%$.
\end{abstract}

Keywords: Sentiment Analysis, Social Network Analysis, Pattern Recognition

\section{Introducción}

Las personas pueden expresar en una publicación de forma indirecta algún tipo de emoción. Identificar estos sentimientos es una tarea sumamente complicada, debido a que se requieren diversos tipos de análisis de morfología, ortografía, sinónimos, entre otros. Este proceso cuando es manual resulta ser complicado y tedioso.

El análisis de sentimientos puede ser combinado con otras técnicas de análisis de redes sociales para identificar usuarios potenciales para recomendar campañas publicitarias, detectar conflictos de interés y aceptación de temas sociales en grupos de usuarios, entre otras aplicaciones.

De acuerdo al estado del arte en análisis de sentimientos, tratar de automatizar este análisis implica al menos los siguientes mecanismos en una arquitectura de software que de solución al problema:

- Extraer texto: Recuperar el texto de interés considerando la complejidad de la arquitectura de datos de las fuentes de información. 
- Normalizar texto: Por medio de un tratamiento lingüístico recuperar únicamente palabras clave que se puedan asociar con emociones, tomando en cuenta polisemia, sinonimia, negaciones de verbos entre otros.

- Clasificar texto: Diseñar mecanismos de reconocimiento de patrones, a fin de alcanzar una mejor precisión al momento de clasificar las publicaciones en las categorías de sentimientos definidas.

En este trabajo, proponemos una de arquitectura de software que considera estos mecanismos para clasificar publicaciones de Facebook en tres emociones: alegría, enojo y tristeza. El sistema esta limitado a las siguientes restricciones de diseño: selección de publicaciones en español, usuarios mexicanos ubicados en la Ciudad de México y zona metropolitana, y se asume que las palabras de las publicaciones se han escrito con ortografía correcta.

Este documento se organizada de la siguiente manera: En la sección II se describen los trabajos relacionados, la sección III muestra la metodología, la sección IV explica los resultados obtenidos, y por último se discuten las conclusiones.

\section{Trabajos relacionados}

Diferentes algoritmos de clasificación han sido probados en análisis de sentimientos en [2], donde se extraen subtítulos de escenas de películas y se identifican aquellas que son emocionales usando clasificadores entrenados. Este enfoque utiliza etiquetas de emociones asociadas a un conjunto de palabras.

Actualmente para el análisis de sentimientos en texto existen corpus como WordNet Affect [3] y Affective Norms for English Words (ANEW) [4], que facilitan usar una estrategia semántica para detectar emociones en texto. Estas estrategias se ha usado en [7] y [8] donde se extrae titulares de noticias asociándolos en seis tipos de emociones con una precisión del $38 \%$.

Estos enfoques han sido combinados con algoritmos de clasificación, en [1] donde se clasificaron mas de 7000 publicaciones de twitter en opiniones positivas, negativas y neutras con una precisión del $61 \%$.

Nuestro trabajo propone un enfoque que combina un clasificador sémantico que consume una jerarquía de palabras enriquecida y un clasificador Nayve Bayes. Los resultados obtenidos respaldan el enfoque propuesto.

\section{Metodología}

Nuestra Arquitectura Web consiste en los siguientes componentes de software: extracción de publicaciones usando el núcleo de la interface de programación de Facebook [9] (Facebook Graph API ), pre-procesamiento de palabras, clasificador base semántico, clasificador Nayve Bayes, funciones de administración web y despliegue de resultados. 


\subsection{Componente de extracción de publicaciones}

El kit de desarrollo de software de Facebook [10] (Faceebook SDK), fue empleado para poder extraer diversos atributos de los usuarios En la tabla 1 se muestran los atributos más importantes:

Tabla 1. Datos de usuario extraídos

\begin{tabular}{|c|c|c|}
\hline Campo & Descripción & Se almacena \\
\hline ID Facebook & Id de usuario. & $\mathrm{Si}$ \\
\hline Género & El género del usuario. & $\mathrm{Si}$ \\
\hline Nombre de usuario & Nombre de usuario en Facebook. & $\mathrm{Si}$ \\
\hline Correo & Email de registro en Facebook. & $\mathrm{Si}$ \\
\hline Publicaciones & Publicaciones realizadas en el perfil. & $\mathrm{Si}$ \\
\hline
\end{tabular}

Las publicaciones son extraídas y almacenadas cuando el usuario decide iniciar el proceso de análisis de sentimientos. El componente extrae las 100 publicaciones más recientes del muro de Facebook del usuario, en la Fig. 1 se muestra parte de la aplicación web usada para la extracción de información.

Posteriormente el texto de las publicaciones es normalizado procesando signos de puntuación (emoticones), filtrando palabras indeseadas (stopwords) y obteniendo la forma canónica de las palabras (lematización).

Secuencias y signos de puntuación: Existen secuencias de signos conocidas como emoticones que describen un contexto emocional: :), :(, :P, $x D, \neg \neg$, entre otros. Por esta razón, los emoticones no son eliminados del texto de la publicación. Los emoticones son empleados particularmente por usuarios jóvenes de Facebook El resto de los signos son eliminados, a excepción de ., ; y ,, porque separan ideas en un texto. En este caso la clasificación final de una publicación que contiene más de una idea, será el resultado promedio de la clasificación individual de cada una de las ideas.

Filtrado de stopwords: Existen palabras que son irrelevantes en el análisis de sentimientos en texto, como preposiciones, artículos, entre otras. Estas palabras son eliminadas de las publicaciones porque pueden generar resultados imprecisos durante la clasificación. La Fig. 2 muestra la visión general del comportamiento de este componente.

Detección de la palabra pero: Generalmente, cuando utilizamos la palabra pero en una oración, estamos explícitamente negando la idea antepuesta a esa palabra, restando relevancia y enfocándonos en la idea posterior. Lo anterior no es una regla infalible, pero se cumple en la mayoría de los casos por lo que se decidió emplearla. 


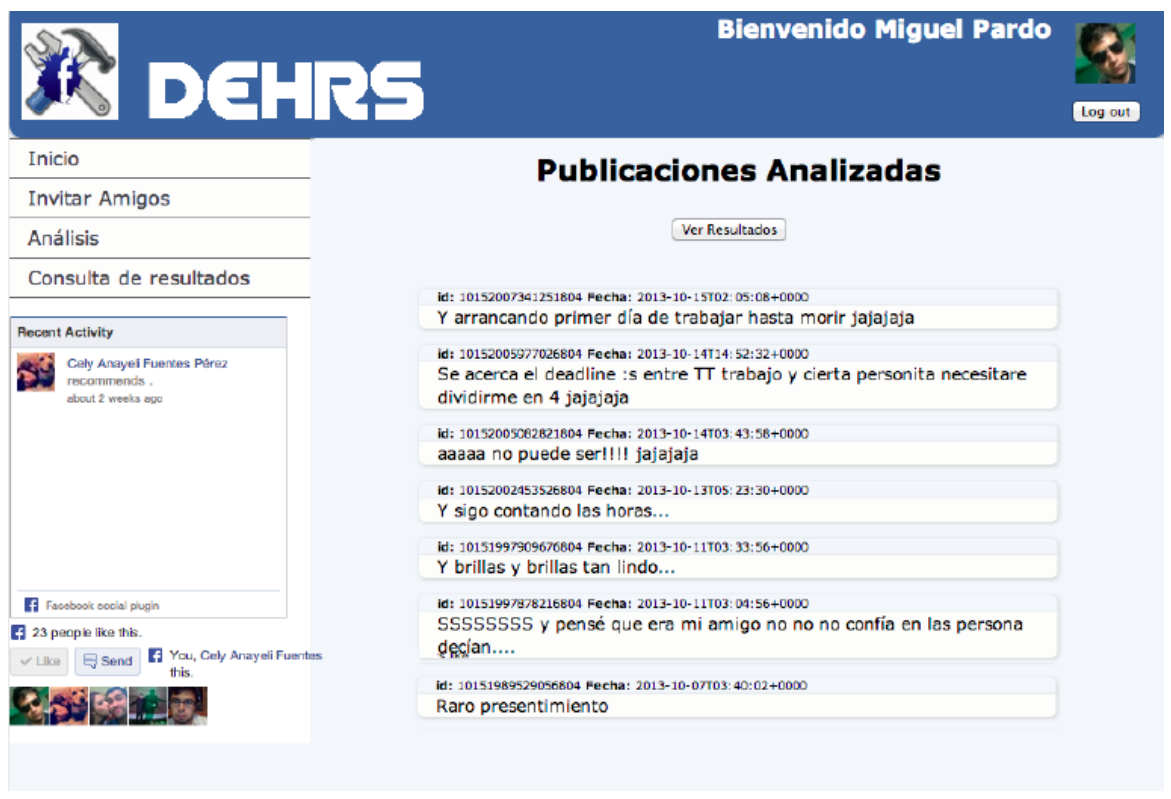

Fig. 1. Interfaz para la extracción de datos

\subsection{Componente para preprocesamiento de texto}

Esta regla resulta ser de gran utilidad en los casos en los que en una misma publicación existen dos o más ideas en las que puede haber, de igual manera, dos o más sentimientos involucrados. Por ejemplo en la publicación: ahora sí me mojé horrible pero me encantó tanto jajaja", la primera idea refleja un sentimiento negativo que se ve anulado por la segunda idea que se encuentra posterior a la palabra "pero". Partiendo de esto, las ideas que precedan a la palabra "pero" son eliminadas, dejando sólo la idea o ideas que se encuentren posterior a esa palabra.

Negaciones: Debido a la naturaleza con la que nos expresamos, existen palabras que pueden cambiar radicalmente el sentimiento que deseamos transmitir, por ejemplo, en la expresión "No estoy feliz", la palabra "no" niega completamente la idea subsiguiente. Estas negaciones son tomadas en cuenta, de manera que si en el texto de la publicación se encuentra el adverbio "no", "nunca" o "ningún", a éste se le concatena un "_" seguido de la siguiente palabra. Y en caso de que no exista otra palabra más en la publicación, la palabra de la negación es eliminada. Para el ejemplo anterior: "No estoy feliz", el resultado de la publicación procesada es "no_feliz" debido a que "estoy" es stopword.

Lematización: Para eliminar las variantes morfológicas de las palabras que componen las publicaciones, se utilizó un lematizador con base en [11], las pala- 


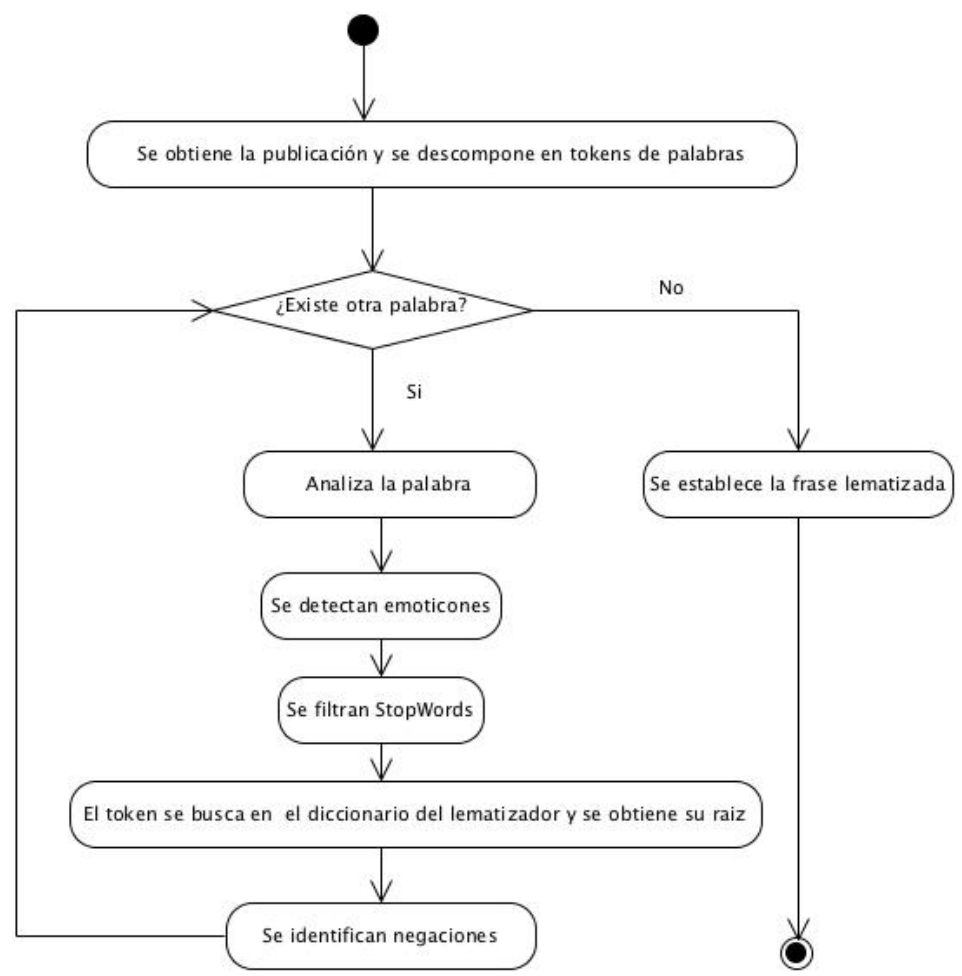

Fig. 2. Fases de ejecución del componente de preprocesamiento de texto

bras que no cuenten con su forma canónica son eliminadas de la publicación y no serán tomadas en cuenta en el clasificador.

\subsection{Componente para clasificación de publicaciones}

En este componente se combinan dos procesos: el primero emplea un recurso que contiene conceptos de sentimientos ponderados basada en WordNet Affect (que se usa como un clasificador base), estos conceptos están organizados y enriquecidos semánticamente como una Ontología. El segundo proceso ejecuta un clasificador Nä̈ve Bayes. Si la publicación no puede asociarse a algún sentimientos, será clasificada como Ambigua.

Clasificador semántico base: El clasificador usa una taxonomía semántica tipo Ontología construida a partir de WordNet-Affect [3]. Esta jerarquía contiene diversos conceptos con carga emocional jerarquizados en diferentes niveles, cada niveles es un grado de intensidad sentimental.

En la tabla 2 se muestra parte de la jerarquía ontológica, la cuál se obtuvo como resultado de una adaptación al español del recurso WordNet Affect que 
involucró el tratamiento de 771 conceptos al español. Estos conceptos están contenidos en una estructura jerárquica, que establece una ponderación asociada que es mayor a medida que el concepto es más cercano a la raíz de un concepto padre que represente una emoción. La estructura jerárquica fue enriquecida agregando a cada concepto sinónimos (que tienen significado emocional) denominados sinónimos directos, también a los conceptos se agregaron expresiones equivalentes conocidas como emoticones porque estos describen estados emocionales. Al no existir corpus oficiales que describan emoticones, se tomaron en cuenta aquellos que son más frecuentes en las publicaciones. Los emoticones seleccionados son expresiones directamente relacionadas con sentimientos, por lo tanto tienen la ponderación más alta.

Este recurso se emplea como un clasificador base, donde cada palabra de una publicación será buscada en la jerarquía. Si la palabra es encontrada, entonces esta recibe un valor que contribuirá numéricamente para decidir si la publicación pertenece a cierta emoción. Por ejemplo, si una publicación contiene la palabra feliz, la cual se encuentra en la jerarquía ontológica con un valor de ponderación 2, entonces esto contribuirá a asociar feliz con el sentimiento alegría, matemáticamente se expresa de la siguiente forma:

$$
\text { Alegría }+=\frac{1}{\text { Ponderación de palabra }}=\frac{1}{2}
$$

La jerarquía consiste en tres conceptos raíz: alegría, enojo y tristeza, a partir de estos existen ramificaciones de conceptos que jerarquicamente describen a estas emociones. Lo que permite que las palabras que están más cercanas a la raíz tengan un peso mayor que aquellas que no lo están, como se muestra en la Fig. 3 y 4 .

El clasificador semántico base en caso de no poder clasificar una publicación en alguno de los tres sentimientos, invoca al clasificador Nä̈ve Bayes

Clasificador Nä̈ve Bayes: Recibe como entrada las palabras normalizadas de una publicación, y mediante un clasificador Naïve Bayes se calcula la probabilidad condicional respecto a cada una de los sentimientos, emplea la siguiente fórmula:

$$
p(\text { Emoción } \mid \text { Publicación })=\frac{p(\text { Emoción }) p\left(\text { palabra }_{i} \mid \text { Emoción }\right)}{\sum_{i=0}^{3} p\left(\text { Emoción }_{i}\right) p\left({\text { Publicación } \left.\mid \text { Emoción }_{i}\right)}\right)}
$$

El denominador de la fórmula (2), también conocido como evidencia, se comporta de manera constante en los cálculos por lo que es omitido, reduciendo la fórmula a:

$$
p(\text { Emoción } \mid \text { Publicación })=p(\text { Emoción })\left(\text { palabra }_{i} \mid \text { Emoción }\right)
$$

Nä̈ve Bayes es un algoritmo de aprendizaje supervisado y fue seleccionado debido a los resultados que ha obtenido en problemas de clasificación de texto según $[6]$. 
Tabla 2. Muestra de conceptos y estructura base de la jerarquía desarrollada.

\begin{tabular}{|c|c|c|c|c|c|c|}
\hline Inglés & Tipo & Traducción & Sinónimos directos & Categoría & Ponderación & Emoción \\
\hline joy & sustantivo & alegría & $\begin{array}{l}\text { júbilo, placer, regoci- } \\
\text { jo, deleite }\end{array}$ & Alegría & 1 & Alegría \\
\hline \begin{tabular}{|l} 
joyfulness \\
\end{tabular} & sustantivo & júbilo & alegría, festividad & Alegría & 1 & Alegría \\
\hline joyful & adjetivo & alegre & $\begin{array}{l}\text { alegre, feliz, agrada- } \\
\text { ble }\end{array}$ & Alegría & 1 & Alegría \\
\hline anger & sustantivo & enojo & $\begin{array}{l}\text { ira, enfado, indigna- } \\
\text { ción }\end{array}$ & Enojo & 1 & Enojo \\
\hline annoyance & sustantivo & molestia & $\begin{array}{l}\text { enojo, fastidio, irrita- } \\
\text { ción, disgusto }\end{array}$ & Molestia & 2 & Enojo \\
\hline chafe & sustantivo & fastidio & roce & Molestia & 2 & Enojo \\
\hline sadden & verbo & entristecer & $\begin{array}{l}\text { apenar, angustiar, } \\
\text { afligir, consternar, } \\
\text { desconsolar, acon- } \\
\text { gojar, amargar, } \\
\text { deprimir }\end{array}$ & Tristeza & 1 & Tristeza \\
\hline
\end{tabular}

Este clasificador se entrenó de forma manual considerando 100 muestras de publicaciones clasificadas, un conjunto de prueba de 802 publicaciones: 227 de Alegría, 202 de Tristeza, 210 de Enojo y 163 clasificadas como Ambiguas. Los resultados que a continuación se describen, argumentan la base experimental de este enfoque

\section{Resultados}

Se realizaron cinco pruebas con una muestra de 802 publicaciones, se combinó diferentes estrategias hasta llegar al enfoque definitivo . Los resultados son los siguientes:

Prueba 1: Usando clasificador base sin procesamiento de emoticones Sin usar los emoticones la presición fue del $17 \%$. Este resultado fue tan bajo debido a que los usuarios de Facebook utilizan palabras coloquiales y emoticones para expresar sus sentimientos. En la muestra usada, se descubrió que 80 de cada 100 publicaciones contienen al menos 1 emoticon. En contraste, al considerar los emoticones la precisión se elevó hasta un $79 \%$.

Prueba 2: Usando solo el clasificador Naive Bayes: La precisión del clasificador con el entrenamiento mencionado en el apartado anterior fue de un $38 \%$.

Prueba 3: Usando clasificador Naive Bayes con filtrado de publicaciones: Para ésta prueba se consideraron todas las muestras al momento de clasificar una nueva publicación, sino únicamente aquellas publicaciones en las que apareciera la palabra en cuestión. Lo anterior ocurre porque se desconoce la cantidad de 


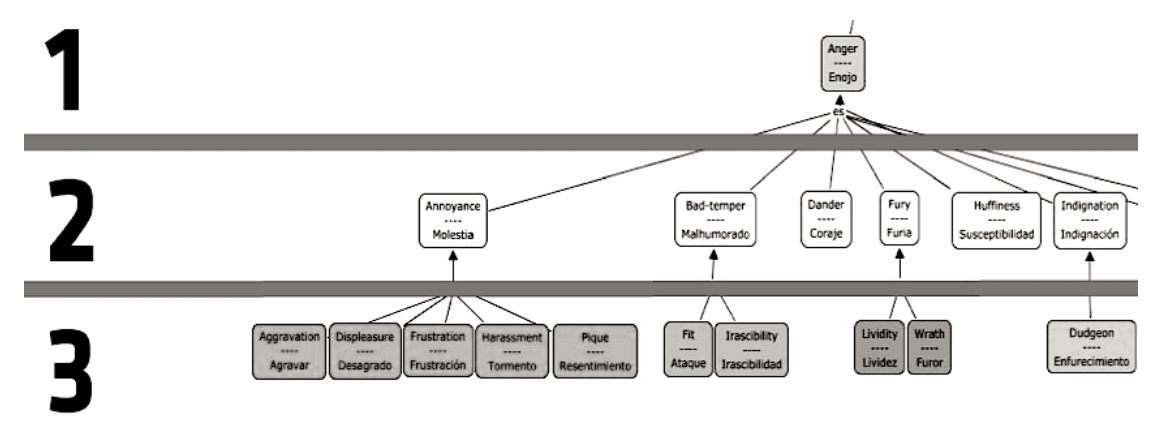

Fig. 3. Primeros tres niveles de ponderación de la jerarquía

palabras que están dentro de las publicaciones asociados aun un sentimiento con respecto a otros. La precisión para esta prueba fue del $44 \%$.

Teniendo en cuenta estos tres resultados la opción es elegir el clasificador semántico basado en la ponderación de la jerarquía. Este clasificador es muy preciso si la publicación a analizar contiene palabras de la jerarquía o algún emoticon.

Debido a esto decidimos realizar otras pruebas pero esta vez combinando los clasificadores anteriores. Dado que el clasificador basado en la ponderación de conceptos es el más preciso se decidió combinarlo con algún clasificador de la prueba 2 o 3 pero sólo en los casos en que las publicaciones no cuenten con palabras de la jerarquía o emoticones, es decir, cuando el clasificador asigne una publicación como ambigua. De esta manera en la jerarquía de conceptos se combina un recurso confiable como WordNet Affect, con un recurso basado en publicaciones que contienen un lenguaje coloquial como lo son las publicaciones de entrenamiento para Naïve Bayes. Las pruebas 4 y 5 son el resultado de esta combinación.

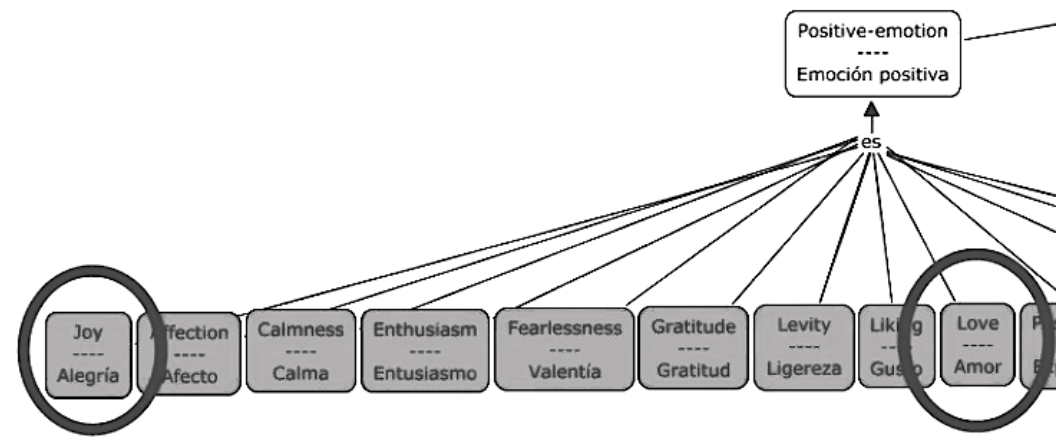

Fig. 4. Conceptos generales que definen alegría y amor 


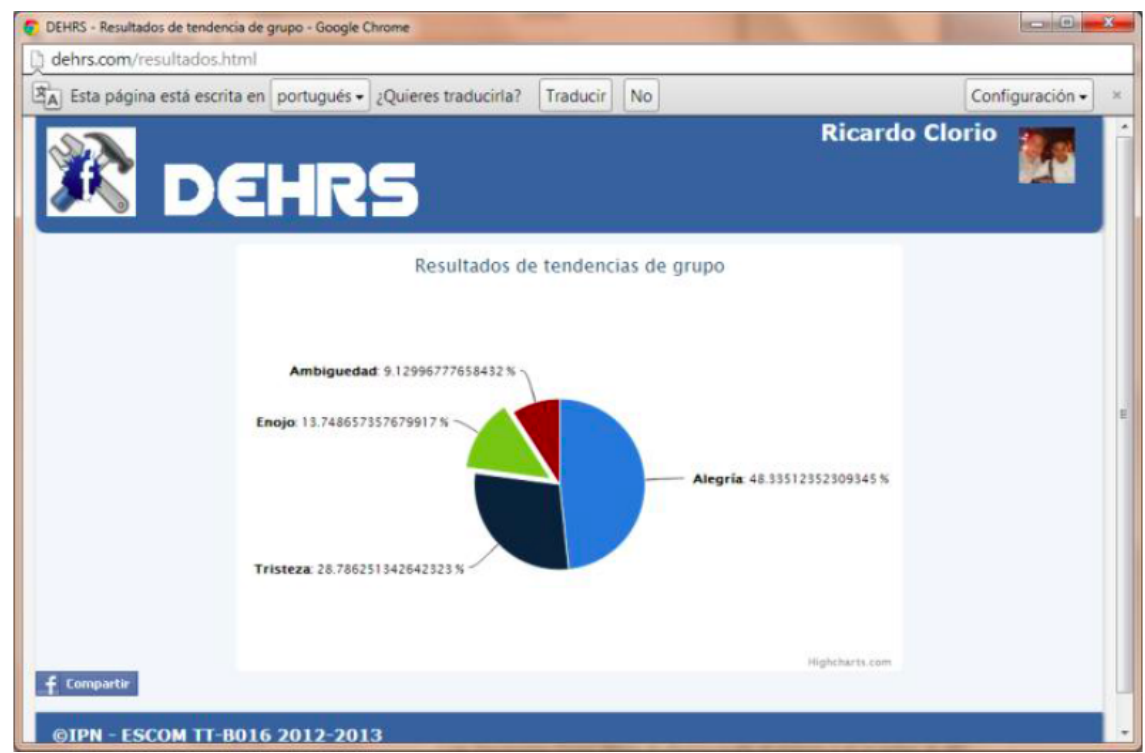

Fig. 5. Despliegue personal de resultados

Prueba 4. Clasificador basado en jerarquía de conceptos y Naive Bayes con filtrado de publicaciones: La precisión de esta combinación fue del $61 \%$. Es evidente que sea una precisión menor a la precisión del clasificador basado en la ponderación de conceptos, debido a que se da pie a clasificar publicaciones que antes sólo eran marcadas como ambiguas, por lo cual da pie a que el resultado de la clasificación sea errónea.

Prueba 5. Clasificador basado en ponderación WNA y Naive Bayes: La combinación de estos clasificadores es la utilizada en la herramienta, su precisión es del $63 \%$. Una de las ventajas de utilizar un algoritmo de aprendizaje supervisado, como Nä̈ve Bayes, es que su precisión va en aumento a medida que más muestras clasifica ya que toma en cuenta todo lo que ha clasificado previamente a la hora de clasificar una nueva muestra.

Finalmente en la Fig. 5 los resultados son mostrados únicamente para las publicaciones personales del usuario que autorizó el análisis, o también se pueden mostrar las tendencias en sentimientos para un grupo de usuarios definidos mediante una consulta realizada por un super usuario del sistema.

\section{Conclusiones}

En este trabajo se presentó una opción útil para análisis de sentimientos en Facebook, combinando un clasificador semántico y un clasificador Nä̈ve Bayes entrenado con publicaciones seleccionadas y que alcanza un desempeño del $63 \%$. 
Sin embargo se detectaron casos donde la clasificación fue ambigua dado que existían palabras relacionadas a más de un concepto contemplado. Por ejemplo, el caso mas común fueron las publicaciones cercanas al concepto "amor".

En el ejemplo anterior, las publicaciones contienen palabras relacionadas a "amor" que también implícitamente estaban asociadas con "alegría", ver Fig. 4. En adición, la jerarquía de conceptos presentada no esta capacitada para detectar dicho concepto, y requiere de un enriquecimiento para contemplar más conceptos asociados a sentimientos.

También es muy importante considerar las características del lenguaje que usa el sector o grupo social que se usará para realizar las pruebas. El uso de emoticones, es común en poblaciones de edad joven y fue un factor decisivo en el diseño de la arquitectura del presente trabajo.

Finalmente es posible mejorar la precisión de clasificación, a través de mecanismos dinámicos que incrementen el número de conceptos asociados a la jerarquía semántica, en combinación de un incremento de los patrones que se usan el entrenamiento del clasificador Naïve Bayes. La aplicación web queda disponible en http://148.204.57.31/dehrs.

\section{Agradecimientos}

Agradecemos el asesoramiento de Miguel Pardo Sixtos, Jesús Morales Alameda y David Ortega Pacheco de la Escuela Superior de Cómputo, así como el soporte económico de la Secretaría de Investigación y Posgrado del Instituto Politécnico Nacional por medio del proyecto multidisciplinario SIP-20130339.

\section{Referencias}

1. G. Sidorov, S. Miranda, F. Viveros, A. Gelbukh, N. Castro, F. Velásquez, I. Díaz, S. Suárez, A. Treviño y J. Gordon, "Empirical Study of Machine Learning Based Approach for Opinion Mining in Tweets" Publicación, Centro de Investigación en Computación, Instituto Politécnico Nacional, México D.F. Septiembre 2012

2. C. Kalyan, Min Y. Kim. "Detecting emotional scenes using Semantic Analysis on Subtitles". Junio 2009. Disponible en: http://nlp.stanford.edu/courses/ cs224n/2009/fp/6.pdf

3. C. Strapparava, A. Valitutti. "Wordnet-affect: an affective extension of wordnet". 2004. Disponible en: http://hnk.ffzg.hr/bibl/lrec2004/pdf/369.pdf

4. Margaret M. Bradley, P. Lang. "Affective Norms for English Words (ANEW): Instruction Manual and Affective Ratings". 1999. Disponible en: http://www .uvm. edu/ pdodds/files/papers/others/1999/bradley1999a.pdf

5. A. Mislove, S. Lehmann, Y. Ahn, J. Onnela, J. Niels. "Pulse of the Nation: U.S. Mood Throughout the Day inferred from Twitter" [online]. 2009. Disponible en: http://www.ccs.neu.edu/home/amislove/twittermood/

6. J. Pekins, "Text Calssification," Python text processing with nltk 2.0 cookbook, L. Subramanian, Ed. Aditya Belpathak, Noviembre de 2010, pp. $167-200$ 
7. C. Strapparava, R. Mihalcea, Annotating and Identifying Emotions in Text, book chapter in Ïntelligent Information Access'", G. Armano, M. de Gemmis, G. Semeraro, and E. Vargiu, Springer, "Studies in Computational Intelligence", 2010.

8. C. Banea, R. Mihalcea, J. Wiebe, Sense-level Subjectivity in a Multilingual Setting, in Proceedings of the IJCNLP workshop on Sentiment Analysis where AI meets Psychology, Chiang Mai, Thailand (2011)

9. Facebook Developers, "Graph API", 2013 Disponible en: https://developers. facebook.com/docs/reference/api/

10. Facebook Developers, "Facebook para sitios web", 2013 Disponible en: https: //developers.facebook.com/docs/guides/web/.

11. Molino Labs, "Lematizador", 2012 Disponible en: http://www.molinolabs.com/ acerca.html\#lematizador 